\title{
The Stability Analysis of Asynchronous Generator Voltage based on SVC
}

\author{
Yaning Zhang ${ }^{a}$, Xinzhen $\mathrm{Wu}^{\mathrm{b}}$ \\ School of Electrical Engineering, Qingdao University, Qingdao 266071, China. \\ azhangyaning68@163.com, bwuxinzhen81@163.com
}

Keywords: Asynchronous generator, SVC, Voltage stability, Reactive power, Simulation.

\begin{abstract}
When the asynchronous generator is running at steady state, inputting load will lead to the voltage drops, and it even cannot meet the requirements of load voltage. In order to maintain the terminal voltage stability of the asynchronous generator, SVC device adopts voltage control strategy to control reactive power. For asynchronous generator running at no load condition stable, the ability had been analyzed that controls the terminal voltage stability of SVC after resistive load was inputted. The good agreement between simulation results and the experimental results verify the validity and accuracy of the method used in this paper.
\end{abstract}

\section{Introduction}

The traditional method of stabilizing the terminal voltage of asynchronous generator, generally, was using switching capacitors [1].But it was unable to compensate dynamically and couldn't maintain a stable terminal voltage, when load changed continuously. Compared with the capacitor compensation, static var compensator (SVC) through power electronics makes reactive power regulation quickly and continuously. Therefore, SVC is suitable for asynchronous generator reactive power compensation to maintain its terminal voltage stabilization [2].

Based on the transient mathematical model of asynchronous generator [3], an asynchronous generator system simulation model has been established, and the structure of thyristor controlled reactors in combination with the fixed capacitors was adopted in this system, which is called the FC-TCR [4]. Voltage closed-loop control strategy is used for the asynchronous generator reactive power compensation, with the same time the capacity of the regulator with resistance load has been analyzed [5].

\section{Asynchronous generator model and the principle of compensation about SVC}

\subsection{Transient mathematical model of asynchronous generator.}

Figure 1 (a) and (b) are the $\alpha$-axis and $\beta$-axis equivalent circuit of asynchronous generator with symmetrical resistive load which are expressed in the stationary coordinate system. In the figure, $R$ is symmetrical resistive load and $C$ is the voltage building capacitance.

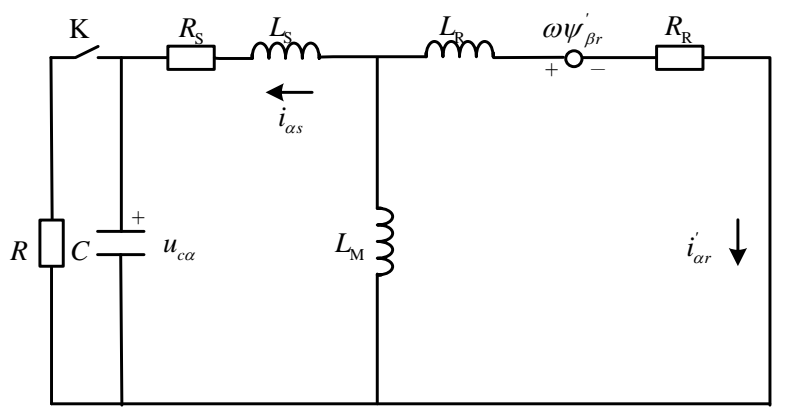

(a) $\alpha$ axis equivalent circuit

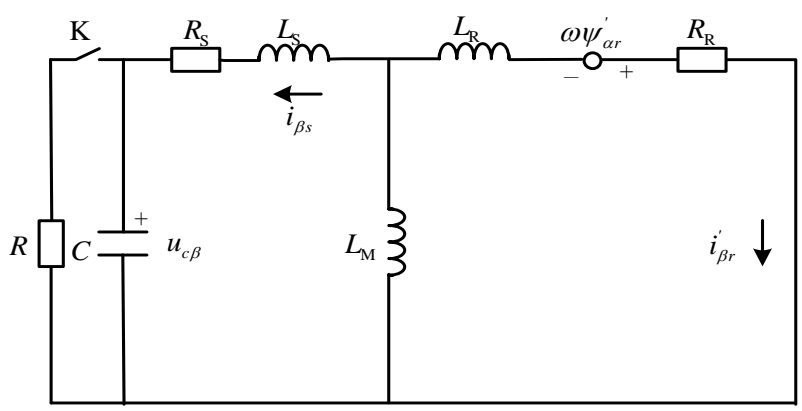

(b) $\beta$ axis equivalent circuit

Fig. 1 Transient equivalent circuit of asynchronous generators under stationary reference frame

According to the reference [6], the state equation of asynchronous generator in the two-phase stationary coordinate system are, namely 


$$
\begin{aligned}
& p \boldsymbol{i}=\boldsymbol{A} \boldsymbol{i}+\boldsymbol{B} \boldsymbol{u} \\
& \boldsymbol{A}=\frac{1}{L_{S} L_{R}-L_{M}^{2}}\left[\begin{array}{cccc}
-R_{S} L_{R} & \omega L_{M}^{2} & R_{R} L_{M} & \omega L_{M} L_{R} \\
-\omega L_{M}^{2} & -R_{S} L_{R} & -\omega L_{M} L_{R} & R_{R} L_{M} \\
R_{S} L_{M} & -\omega L_{M} L_{S} & -R_{R} L_{S} & -\omega L_{S} L_{R} \\
\omega L_{M} L_{S} & R_{S} L_{M} & \omega L_{S} L_{R} & -R_{R} L_{S}
\end{array}\right] \\
& \boldsymbol{B}=\frac{1}{L_{S} L_{R}-L_{M}^{2}}\left[\begin{array}{cc}
L_{R} & 0 \\
0 & L_{R} \\
-L_{M} & 0 \\
0 & -L_{M}
\end{array}\right] \\
& \boldsymbol{i}=\left[\begin{array}{llll}
i_{\alpha 1} & i_{\beta 1} & i_{\alpha 2} & i_{\beta 2}
\end{array}\right]^{T} \\
& \boldsymbol{u}=\left[\begin{array}{ll}
u_{\alpha 1} & u_{\beta 1}
\end{array}\right]^{T}
\end{aligned}
$$

Where $i_{\alpha 1}, i_{\beta 1}, i_{\alpha 2}, i_{\beta 2}$ - rotor currents under two-phase stationary coordinate system determined (A); $u_{\alpha 1}, u_{\beta 1}$ - stator voltages $(\mathrm{V}) ; R_{S}, R_{R}$ - equivalent resistances of stator and rotor $(\Omega) ; L_{S}, L_{R}$ equivalent inductances of stator and rotor $(\mathrm{H}) ; L_{M}$ - equivalent mutual inductance between stator and rotor $(\mathrm{H}) ; \omega$ - angular velocity of rotor $(\mathrm{rad} / \mathrm{s})$.

\subsection{Compensation principle of SVC.}

One-phase connection diagram of three-phase asynchronous generator which runs with symmetrical load and SVC is shown in figure 2

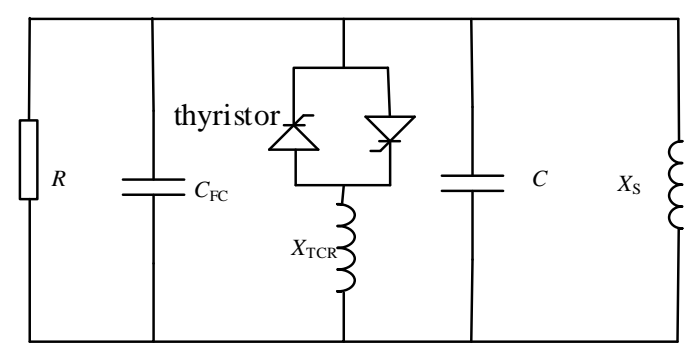

Fig. 2 One-phase diagram of the asynchronous generator with three phase symmetrical load

In Figure 2, $R$ is the load resistance; $C_{\mathrm{FC}}, X_{\mathrm{TCR}}$ are equivalent capacitive reactance value of SVC and equivalent reactance value of TCR; $C$ is the initial voltage building capacitance. We can transform SVC and voltage capacitor to an equivalent capacity $C_{\text {equ }}$, and the formula for the equivalent capacitance $C_{\text {equ }}$ is

$$
C_{\text {equ }}=C_{\mathrm{FC}}+C-\frac{1}{\omega_{1} X_{\mathrm{TCR}}}
$$

From (6) we know that, since $C_{\mathrm{FC}}, C$ are fixed value, so we can change the value of $C_{\text {equ }}$ by adjusting reactance $X_{\mathrm{TCR}}$, and then reactive power is compensated.

\section{Control strategy of SVC}

Under the assumption that the terminal voltage unchanged, in order to maintain the terminal voltage at constant when load changes, we can change the connected in parallel with equivalent susceptance of SVC, thus the total susceptance of the system unchanged, so SVC can maintains the stability of the terminal voltage.

The relationship between fundamental components of the TCR current and the trigger angle of the thyristor is

$$
I_{1}=\frac{2 \pi-2 \alpha+\sin 2 \alpha}{\pi X_{\mathrm{L}}} U
$$

Then the equivalent susceptance of TCR is

$$
B_{\mathrm{L}}=\frac{2 \pi-2 \alpha+\sin 2 \alpha}{\pi X_{L}}
$$


Where $U$-- System voltage; $X_{\mathrm{L}}$-- the inductance value of thyristor series reactance; $\alpha$-- the trigger angle of the thyristor

Based on the above analysis, SVC closed loop control system is shown in Figure 3.

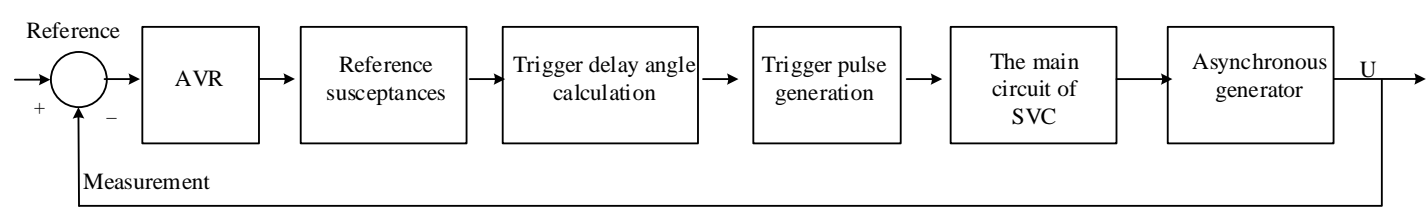

Fig. 3 SVC closed-loop control system diagram

Assume that AVR uses PI control strategy, then the SVC susceptance reference value is determined by the following formula, namely

$$
B_{\text {ref }}=k_{\mathrm{P}} \Delta U+k_{\mathrm{I}} \int \Delta U d t
$$

After the AVR's output goes through the limiter links it will produce the desired reference value of SVC's susceptance, and as long as asynchronous generator terminal voltage reference is given, the entire device automatically adjusts the SVC's susceptance according to the terminal voltage measurement so that the actual terminal voltage will equal to the reference terminal voltage. Then it reaches adjusting the terminal voltage purpose.

\section{Comparative analysis of the simulation results and experimental results}

\subsection{Simulation and experiment.}

Simulation data is saved to Matlab workspace by the oscilloscope, and then the data is processed through corresponding plotting functions. Using a digital recorder instrument records the experimental voltage and current waveforms, and the data is processesed to waveforms.

\subsection{Comparative Analysis of Results.}

The simulation and experimental parameters: input angular velocity is $314 \mathrm{rad} / \mathrm{s}, C$ is $40 \mu \mathrm{F}$, TCR branch of delta connection whose single-phase inductance value is $0.5 \mathrm{H}$, TC Branch Y-shaped connection whose single-phase capacitor value is $40 \mu \mathrm{F}$, voltage reference value of SVC system is $200 \mathrm{~V}$, the load resistance is $200 \Omega$.

Figure 4 (a), (b) are respectively the transient stator voltage simulation waveforms and experimental waveforms, when asynchronous generator is running with SVC from no load steady-state operation to $200 \Omega$ resistive load. Figure 5 (a), (b), respectively, are current waveforms of $C$ branches. When the asynchronous generator with SVC at no-load status is running at stable operation, then resistive load is inputted. The value of terminal voltage begins gradually to decrease from steady-state operation. Due to the regulation of the SVC, the simulation waveform of transient voltage after about $0.5 \mathrm{~s}$ adjustment process, stabilizes at voltage reference value; and the experimental transient voltage after about 1 s adjustment process, stabilizes at voltage reference value; the current of $C$ branch goes into a sudden drop when the load is inputted suddenly, and then with the adjustment of terminal voltage it makes the appropriate changes, until it achieves stability ultimately.

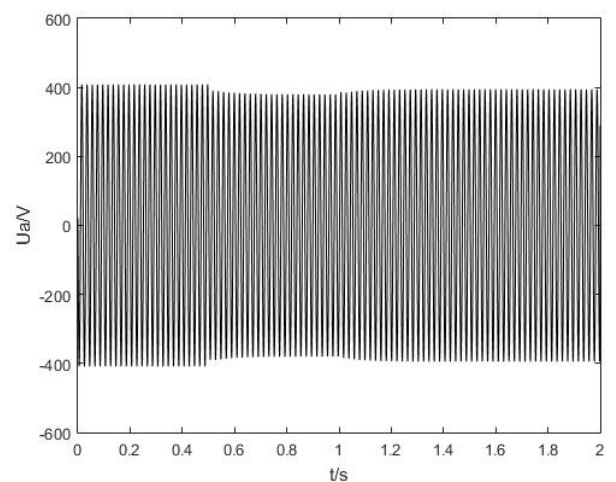

(a) Voltage simulation waveform

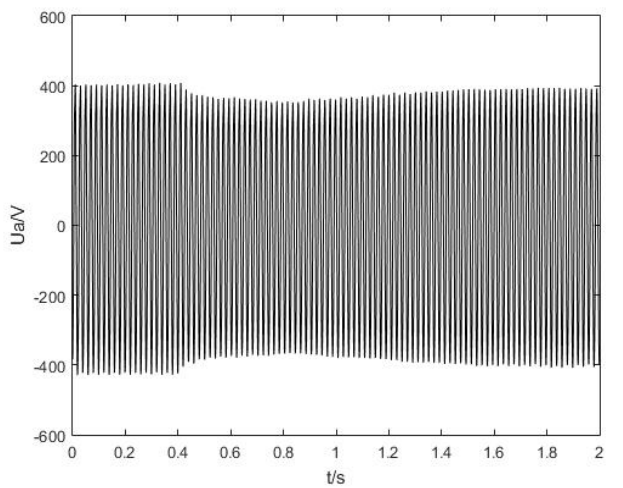

(b) Voltage experiment waveform

Fig. 4 Load and SVC voltage waveform 


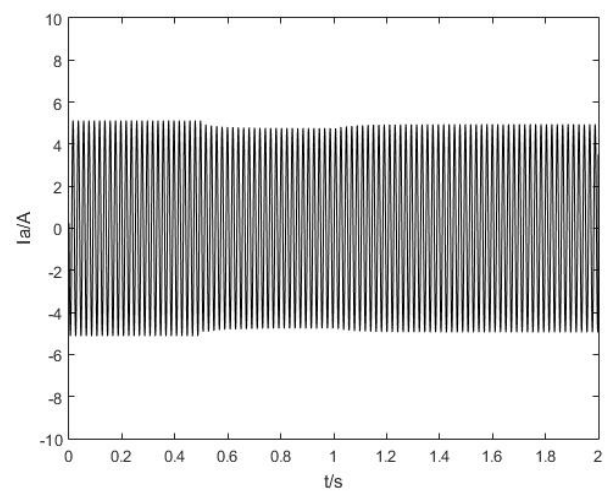

(a) Current simulation waveform

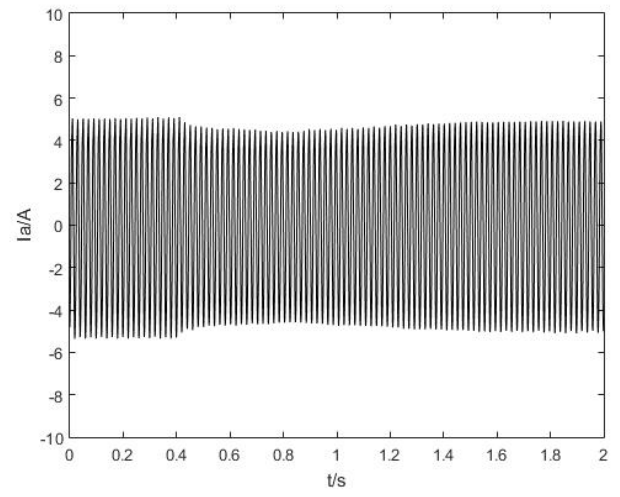

(b) Current experiment waveform

Fig. 5 Load and SVC current waveform

As that can be seen from Fig. 4 and 5, the simulation waveform is ideal, its adjusting time is short, it has well stability, the transient process of it is almost no fluctuation; however, experimental waveforms have longer adjustment time, its stability slightly is worse, the transient process has a slight fluctuation, but they have the same capacity to stabilize voltage eventually, thereby that verify the feasibility and effectiveness of SVC voltage regulator method.

\section{Summary}

A simulation model of asynchronous generator is built through its transient mathematical model. Comparative analysis simulation and experimental results verify the effectiveness of method which is taken by SVC to stable voltage.

The simulation and experimental results show that the use of SVC enables asynchronous generator terminal voltage stability, proof the validity and effectiveness about SVC control strategy and asynchronous generator model which is established in this paper.

\section{References}

[1]. Hao Kuansheng, Wu Xinzhen. Capacitance calculation for keeping constant voltage of asynchronous generators based on transient model. Proceedings of the Chinese Society for Electrical Engineering. (2007) No. 7, p. 51-56.

[2]. Wang Bing. The reactive power compensation calculation and simulation analysis on SVC-based control strategy for urban distribution network. China Electric Power (Technology Edition). Vol. 7 (2013) No. 2, p. 32-35.

[3]. Bhim Singh. A Stand-Alone Generating System Using Self-Excited Induction Generators in the Extraction of Petroleum Products [J]. IEEE Trans. on Industry Applications, 2010, 46(1):94-101(in Chinese).

[4]. Wang Zhaoan, Yang Jun, Liu Jinjun, Wang Yue. Harmonic suppression and reactive power compensation. Machinery Industry Press, 2005, p. 175-189.

[5]. Xu Weiqing, Hao Kuansheng, Lan Yong, Liu Yaohua. Research on constant voltage operation of self-excited asynchronous generator with resistance loads. Marine Electric \& Electronic Technology. Vol. 26 (2006) No. 4, p. 102-105.

[6]. Wang L., Su J.y. Dynamic performances of an isolated self-excited asynchronous generator under various loading conditions. IEEE Trans. on EC. Vol. 14 (1999) No. 1, p. 93-100. 